\title{
Inverse cross-modality matching: A test of ratio judgment consistency for group and individual data
}

\author{
MICHAEL G. LILIENTHAL and WILLIAM E. DAWSON \\ University of Notre Dame, Notre Dame, Indiana 46556
}

\begin{abstract}
Direct and inverse cross-modality matches made by 20 subjects were assessed for ratio judgment consistency. Each subject matched apparent duration to loudness, and vice versa, in both a directly and an inversely proportional manner. All four tasks were repeated twice so that individual differences could be examined using interrepetition correlations. Group data exhibited the appropriate inverse relationship indicative of consistency, although the inverse matches were slightly curvilinear and resembled earlier studies with inverse attribute scales. Some individuals showed a high degree of consistency but many departed widely from inverse proportionality. Individual differences in exponents, which occurred for both types of tasks, were not removed by S. S. Stevens' (1971) regression balance procedure. However, interrepetition correlations for the differences in the absolute values of the direct and inverse exponents of individuals were nonsignificant, suggesting that when subjects' exponents differed for the two types of tasks they did so on a random basis. The latter finding implies that subjects would give inversely proportional matches were it not for random factors. The findings were discussed in relation to other types of ratio consistency.
\end{abstract}

S. S. Stevens (1957) has argued that subjective magnitude grows as a power function of the physical intensity of a stimulus, i.e., $\Psi=K \Phi^{n}$, where $\Psi$ is the subjective magnitude, $K$ equals a constant which is dependent on the units used, $\Phi$ is the physical intensity, and $n$ is some power which is appropriate to the modality. The power law has been verified for group data with many sensory continua such as loudness, brightness, duration, vocal effort, handgrip, and taste (S. S. Stevens, 1957, 1961). It implies that equal stimulus ratios produce equal sensation ratios. When a subject makes a numerical estimation, as in magnitude estimation, or adjusts some continuum, as in cross-modality matching, he is supposedly indicating ratio relationships. That is, the subject supposedly matches numbers or the continuum under his control in an appropriately proportional manner. If subjects are indeed indicating subjective ratios properly in such tasks, they would be expected to show consistency when instructed to manipulate the ratios in a slightly different manner.

Cliff (1973) has stated that ". . . meaningful scaling takes place when several ingredients exist. The first of these is a set of data relations which are at least fairly consistent and moderately numerous. A second requirement is the existence of a mathematical model

Based on a master's thesis completed by the first author at the University of Notre Dame. Also presented as a paper at the meeting of the Midwestern Psychological Association, Chicago, May 1975. The authors thank Reverend William A. Botzum for his assistance with some of the statistical analysis. Requests for reprints should be sent to William E. Dawson, Department of Psychology, University of Notre Dame, Notre Dame, Indiana 46556. whose variables can be matched with the empirical conditions and the relations among them." Stevens has satisfied Cliff"s second requirement, in that his model is empirically testable and it leads to explicit scale values. But are the data relations, which are expected from the power law, consistent? In this study, the consistency question was examined by means of a comparison of the intensities subjects produced in a cross-modality matching task with those produced in an inverse cross-modality matching task, where they matched one continuum to another in an inverse manner. In typical cross-modality matching, subjects match the strength or magnitude of sensations from two different modalities. In inverse cross-modality matching, the procedure is similar but with one important difference: the subject makes an inversely proportional match between continua. Thus, for instance, a subject asked to match time duration to a presented line length would produce a duration that appeared one-fourth as great if he perceived the line as four times longer.

The procedure is similar, but not equivalent, to that used to obtain inverse magnitude scales (e.g., S. S. Stevens \& Guirao, 1962; Torgerson, 1960). In the latter task, subjects are instructed to judge an inverse attribute of the stimulus; the present procedure requires the matching of the same attribute but in an inverse manner. Inverse scales have been found to be approximate inverses of their corresponding magnitude scales, but the relation has tended toward nonlinearity on log-log axes (John, 1971; Torgerson, 1960). The present study was interested in, among other things, whether the inverse relation would be more precise with the inverse matching method. 
Mathematically, the inverse cross-modality matching task is represented by a match between $\Psi_{1}^{-1}$ and $\Psi_{2}$. where $\Psi_{1}=k_{1} \Phi_{1}{ }^{n}$ and $\Psi_{2}=k_{2} \Phi_{2} m$. Thus, if subjects make consistent matches, then $\left(k_{1} \Phi_{1} n\right)^{-1}$ should equal $k_{2} \Phi_{2} m$. Further manipulation gives

$$
\Phi_{2}=B \Phi_{1}(\text { inv } \mathrm{cmm})^{-\mathrm{n} / \mathrm{m}},
$$

where $B$ is a new constant. In the usual cross-modality match.

$$
\Phi_{2}=C \Phi_{1}(\mathrm{cmm})^{\mathrm{n} / \mathrm{m}}
$$

where $\mathrm{C}$ is also a constant (S. S. Stevens, 1961). Equations 1 and 2 taken together give

$$
\Phi_{1}(\text { inv cmm })=D \Phi_{1}(\mathrm{cmm})^{-1} \text {. }
$$

where $D=(C / B)^{-m / n}$. Equation 3 implies that the plot of the adjustments for an inverse match against those of the ordinary match should produce a straight line with a slope of -1.00 in $\log -\log$ axes. Such a straight line would be evidence that subjects are consistent in their ratio judgments across the two methods. In addition, it would offer additional support for the validity of Stevens' law.

A question that has been asked about subjects' performance with direct scaling techniques is: Does the power function hold for the data of individual subjects? Individual functions have been found to follow Stevens power law for the methods of magnitude estimation and magnitude production (J. C. Stevens \& Mack. 1959) and for cross-modality matching (Wanschura \& Dawson, 1974). The present study tried to determine whether this also holds true for inverse matching scales. Further, the results for individuals were examined to see if the predicted inverse relationship held.

Although direct scaling data for individuals have followed the power function, the exponents obtained have varied widely across individuals. Evidence for reliable interindividual variation has occurred with ratio estimation (Ekman, Hosman, Lindman, Ljungberg, \& Akesson, 1968), magnitude estimation (Jones \& Marcus, 1961), cross-modality matching (Rule \& Markley, 1971), and sensory-modality matching (Dawson \& Mirando, 1975). To determine whether such individual differences occur with the inverse matching task, each task was carried out twice and the interrepetition correlation approach of Rule (1966) was used for detection of persisting differences. In addition, a regression balance (S. S. Stevens \& Greenbaum, 1966) was planned in an attempt to reduce the amount of intersubject variability. S. S. Stevens (1971) suggested that a reduction is apt to occur with such a balance, but Wanschura and Dawson (1974) found little, if any, such a decrease for cross-modality matches.
To summarize, the present study endeavored to answer the following questions: Are the group results of an inverse cross-modality match inversely related in the proper manner to the results of a standard cross-modality match? Such a finding would be expected if subjects can indeed judge sensory ratios in a consistent manner, and if Stevens' power law is correct. Do the data for individual subjects in the inverse matching task show this expected consistency? Do individual differences in exponents occur in the inverse scaling task? If so, does a regression balance eliminate this effect?

\section{METHOD}

\section{Selojects}

Five female and 15 male naive undergraduate volunteers from introductory psychology classes at the University of Notre Dame took part in the experiment. The subjects received credit in their courses for their participation.

\section{Apparatus}

Two key-operated switches were used by subjects to enable them to control the presentation of the loudnesses and the time durations. For the matching of binaural loudness to time duration, $1,000-\mathrm{Hz}$ tones were produced by an audio oscillator whose output level was controlled by a variable attenuator and sone potentiometer (Poulton \& S. S. Stevens, 1955) connected to a pair of calibrated earphones. The earphones were calibrated so that the voltage measured across them could be converted to sound pressure measured in dynes $/ \mathrm{cm}^{2}$. The time durations were controlled by a Hunter timer, which lit a small white lamp for the appropriate interval. The time intervals employed were $0.30,0.60,1.20,2.40$, 4.80 , and $9.60 \mathrm{sec}$

For the matching of duration to loudness, subjects controlled the time duration by depressing the key which lit the lamp for the desired time interval. The duration was recorded by a Standard timer. The $1,000-\mathrm{Hz}$ tones to be matched were presented binaurally and included sound pressure levels of $40,50,60,70,80$, and $90 \mathrm{~dB}$ (re $20 \mu \mathrm{N} / \mathbf{M}^{2}$ ).

\section{Procedure}

The subjects took part one at a time. They were asked to carry out four tasks, all of which were done in the first two sessions and then repeated in the last two sessions using an appropriately counterbalanced order. For one task, the subjects performed regular cross-modality match in which they adjusted the perceived loudness of a tone to match perceived time duration. All six intervals were presented to each subject twice in an irregular order but with all six presented before any one was repeated. Another task involved the cross-modality matching of duration to loudness with each of the six tones presented twice in a similar irregular order. For the remaining two tasks, the subjects carried out an inverse cross-modality match of loudness to duration and then of duration to loudness. Stimulus presentation was similar to that for the other two tasks.

Instructions for cross-modality matching were those typically given for such a task; the instructions for the inverse matching of loudness to duration were as follows: "You will be presented with a series of time durations indicated by how long the white lamp in front of you stays lit. Your task is to set the loudness level by means of the dial so that it is the inverse or opposite of your subjective impression of the duration presented. Assign the first time duration any loud ness that seems appropriate. For successive durations. set the loud ness proportionately in an inverse manner. For instance, if a duration is 5 times as long as the first one, set the loudness $1 / 5$ as loud as the first. If the duration is $1 / 20$ as long as the first, set the 
loudness $\mathbf{2 0}$ times as loud as the first. In adjusting the loudness. I want you to go above and below the range that seems right and eventually zero in on the point that seems right. At no time should you try to time the durations by counting, holding your breath, or the like. Just make your matches agree with your casual impression of the time durations. In this experiment, there are no right or wrong answers. Any questions?" The instructions given to the subjects for the inverse matching of duration to loudness were similar but with the appropriate word changes.

In the first session, half of the subjects performed both cross-modality tasks and the other half performed both inverse tasks. In the second session, subjects performed the remaining two tasks. Within each of the halves, half began by adjusting loudness and the other half by adjusting duration. The third and the fourth sessions involved repetitions of the tasks in the first two but with the order of the tasks counterbalanced. The intersession interval for any subject was always greater than $24 \mathrm{~h}$ but never more than 2 weeks in length.

\section{RESULTS}

\section{Group Data}

Geometric means of the adjustments were calculated for the durations matched to each sound pressure level and for the sound pressures matched to each time interval. They were computed for the adjustments pooled over subjects and repetitions for each cross-modality match and for each inverse match and. also, for these tasks for each repetition pooled over subjects. Next, power functions were fitted to the geometric means for the various combinations of tasks and repetitions; the resultant exponents are reported in Table 1. Regression balances were obtained by taking the geometric mean of the duration exponent and the corresponding reciprocal of the loudness exponent. The resulting balanced exponents range from .52 to .57 in absolute size. Regression angles. measured in radians, are also listed in Table 1 . The values of the regression angles and the various exponents in this table are approximately the same as found by Wanschura and Dawson (1974) using the same two sensory continua. Further, they are similar in magnitude for both the direct matches and the inverse matches, a new finding of the present study.

The exponents for cross-modality tasks have been predicted previously by experimenters using the exponents from magnitude estimation tasks. S. S. Stevens (1961) placed the exponent for duration at 1.10. and more recently it has been determined at 1.03 (S. S. Stevens \& Greenbaum, 1966). The

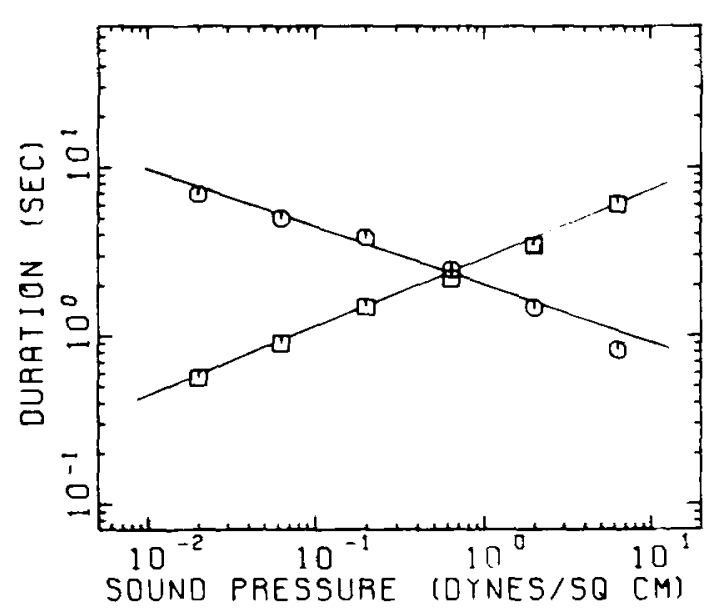

Figure 1. Geometric means of the cross-modality matches (squares) and inverse cross-modality matches (octagons) of apparent duration to loudness for data pooled over repetitions and subjects.

loudness exponent has been reported anywhere between .60 (S. S. Stevens, 1955) and .68 (S. S. Stevens, 1969). Thus, the predicted slope in a log-log plot for sound pressure as a function of time lies within the interval $.60 / 1.10$, or .55 , and $.68 / 1.03$, or .66 . The exponents for duration matched to loudness are much lower than predicted, but this is probably due to the "regression effect" (S. S. Stevens \& Greenbaum. 1966). The regression balanced exponents lie much closer to the predicted interval. The present data show that the balanced exponent for an inverse cross-modality match also falls close to the predicted range.

Statistical tests revealed that the repetitions variable did not significantly affect the exponents obtained and did not interact with other variables; therefore subsequent analysis was largely restricted to the combined repetitions data. Figure 1 is a plot of the geometric means of the durations produced in the cross-modality and inverse matches to loudness; similarly, Figure 2 presents the results of the corresponding matches to duration. The straight lines in these figures were fitted according to the criterion of least squares. Approximately $99 \%$ of the variability in the cross-modality tasks is accounted for by the fitted line, while $98 \%$ is accounted for in the inverse matching tasks.

Table 1

Exponents, Regression Angles, and Balanced Exponents for Data Pooled Across Subjects

\begin{tabular}{|c|c|c|c|c|c|c|}
\hline $\begin{array}{c}\text { Matching } \\
\text { Task }\end{array}$ & Repetition & $\begin{array}{l}\text { Duration } \\
\text { Adjusted }\end{array}$ & $\begin{array}{c}\text { Loudness } \\
\text { Adjusted }\end{array}$ & $\begin{array}{l}\text { Reciprocal } \\
\text { Loudness }\end{array}$ & $\begin{array}{c}\text { Regression } \\
\text { Angle }\end{array}$ & $\begin{array}{l}\text { Balanced } \\
\text { Exponent }\end{array}$ \\
\hline Direct & $\begin{array}{c}1 \\
2 \\
1 \text { and } 2\end{array}$ & $\begin{array}{l}.39 \\
.42 \\
.40\end{array}$ & $\begin{array}{l}1.32 \\
1.30 \\
1.31\end{array}$ & $\begin{array}{l}.76 \\
.77 \\
.76\end{array}$ & $\begin{array}{l}.28 \\
.26 \\
.27\end{array}$ & $\begin{array}{l}.54 \\
.57 \\
.55\end{array}$ \\
\hline Inverse & $\begin{array}{c}1 \\
2 \\
1 \text { and } 2\end{array}$ & $\begin{array}{l}-.35 \\
-.38 \\
-.37\end{array}$ & $\begin{array}{l}-1.29 \\
-1.21 \\
-1.25\end{array}$ & $\begin{array}{r}-.78 \\
-.83 \\
-.80 \\
\end{array}$ & $\begin{array}{l}.33 \\
.33 \\
.32\end{array}$ & $\begin{array}{l}-.52 \\
-.56 \\
-.54 \\
\end{array}$ \\
\hline
\end{tabular}




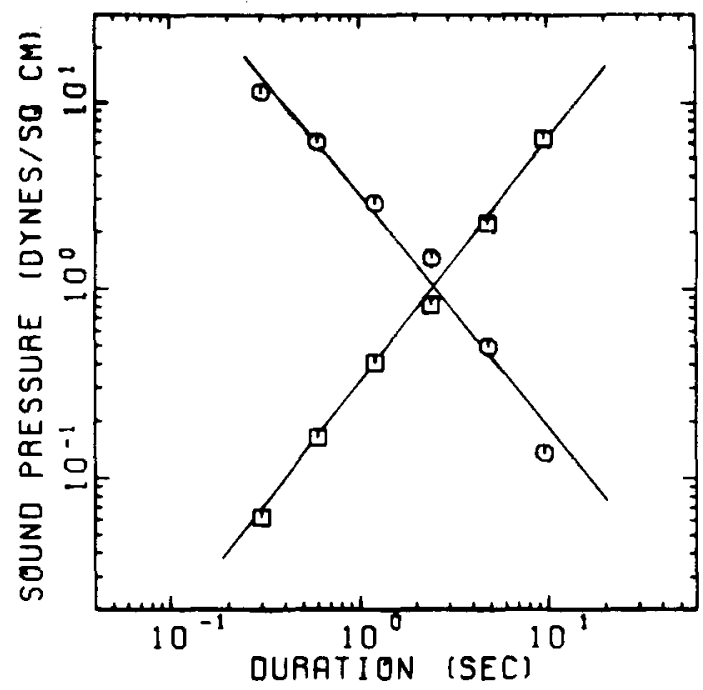

Figure 2. Geometric meane of crose-modality matches (squares) and burene crem-modality matches (octagom) of apparent budnes: to duration for data pooled over repetitions and eabjects.

Although the fits are good, the inverse matching data, unlike the direct matching data which appear very linear, seem to be curvilinear. As a result, trend analyses were carried out to assess the linearity of all of the data. For direct matching, there was a highly significant linear trend with $F(1,19)=187.39$, $\mathrm{p}<.001$, and $F(1,19)=165.34, \mathrm{p}<.001$, for duration and loudness adjustments, respectively. These trends each accounted for $99.7 \%$ of the overall variance. When $F$ tests were performed for the residual trends, it was found that the components beyond the linear when combined did not contribute significantly to the shape of the curve. For loudness adjustment, $F(4,19)=.45$, and for duration, $F(4,19)$ $=.37$.

For the inverse matches using duration, a linear trend, $F(1,19)=198.95, p<.001$, accounted for $97.9 \%$ of the variability. As with the direct matches, the residual trend was nonsignificant, $F(4.19)=1.69$, $p<.25$. In the case of the inverse matches with loudness, both the linear trend, $F(1,19)=168.82$, $p<.001$, and the quadratic trend, $F(1,19)=19.25$, $\mathrm{p}<.01$, were significant. The linear component contributed $97.9 \%$ of the variability, while the quadratic involved only $1.9 \%$. Thus, although the data for the inverse tasks appear somewhat curvilinear in Figures 1 and 2 , only the inverse loudness quadratic component is statistically significant.

\section{Consistency in Group Exponents}

The consistency of the ratio judgments by subjects, indicated by the degree to which the slope of the line produced by plotting inverse matches as a function of direct matches approaches -1.0 , can be assessed in Figure 3 . The slopes are approximately -1.0 , but the data are not perfectly linear-a fact that follows from the tendency toward nonlinearity in the inverse matching results. Least squares estimates of the two slopes were obtained using a procedure described by Kenney (1939). It finds the best fitting straight line for data pairs for the case where both variables are subject to error. The fitted slopes were -0.91 for duration adjustment and -0.96 for the adjustment of loudness. Each line accounts for $98 \%$ of the variability of the data as plotted. Neither slope was found to differ significantly from -1.0 when $t$ tests were conducted using the exponents for individuals in Table 4. Since geometric means of adjustment were employed as averages, the slopes of the best-fitting lines for group data do not equal the arithmetic means of the slopes fur individuals given in Table 4. The latter means are even closer to -1.0 .

\section{Individual Exponents}

The power function exponents of each subject for the various tasks were also calculated. Again, a power function provided a fairly good fit for the individual data. The straight lines accounted for $66 \%$ to $80 \%$ of the variability, on the average, for an individual subject depending on the task. The quality of the individual functions resembled that found in Wanschura and Dawson (1974). It is interesting to note that subjects who tended to have a high exponent in the direct matches also had high exponents (but negative) for the inverse matches. Likewise, many subjects with low exponents in the direct tasks also had low exponents for the inverse tasks. This trend

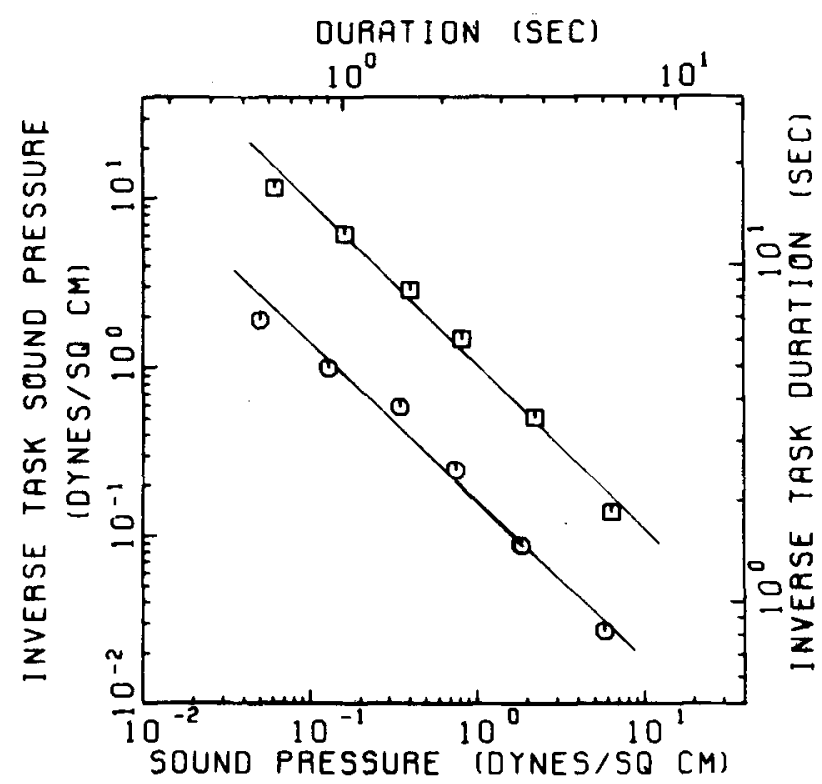

Figure 3. Inverse croes-modality matches of apparent boutheas (octagons) and apparent duration (equares) as a function of the apparent londneas and apparent duration, respectlvely, in crose-modality matches for data pooled over repettition and subjects. 
Table 2

Exponents for Individuals for Each Task and Modality Pooled Over Repetitions

\begin{tabular}{ccccc}
\hline & \multicolumn{2}{c}{$\begin{array}{c}\text { Duration Adjusted } \\
\text { CMM }\end{array}$} & \multicolumn{2}{c}{ Loudness Adjusted } \\
CMM-1 & \multicolumn{2}{c}{ CMM } & CMM-1 \\
\hline 1 & .64 & -.57 & 1.11 & -.95 \\
2 & .22 & -.17 & .76 & -1.02 \\
3 & .41 & -.36 & 1.18 & -1.29 \\
4 & .43 & -.35 & 1.80 & -1.80 \\
5 & .43 & -.37 & 1.36 & -1.31 \\
6 & .40 & -.37 & .81 & -.72 \\
7 & .54 & -.62 & 1.94 & -1.35 \\
8 & .17 & -.27 & 1.77 & -2.30 \\
9 & .29 & -.29 & 1.54 & -.86 \\
10 & .47 & -.38 & 1.13 & -1.12 \\
11 & .60 & -.50 & 1.82 & -1.27 \\
12 & .37 & -.41 & .53 & -.41 \\
13 & .35 & -.36 & 1.22 & -1.01 \\
14 & .51 & -.31 & 1.24 & -1.17 \\
15 & .39 & -.35 & 1.80 & -1.89 \\
16 & .19 & -.16 & 1.07 & -1.35 \\
17 & .38 & -.29 & 1.53 & -1.44 \\
18 & .25 & -.34 & 1.98 & -1.78 \\
19 & .49 & -.33 & 1.03 & -1.02 \\
20 & .53 & -.51 & .52 & -.94 \\
Mean & .403 & -.365 & 1.307 & -1.249 \\
SD & .132 & .116 & .454 & .440 \\
\hline
\end{tabular}

Table 3

Correlations Comparing Cross-Modality Matching and Inverse Cross-Modality Matching Exponents for Single and Combined Repetitions

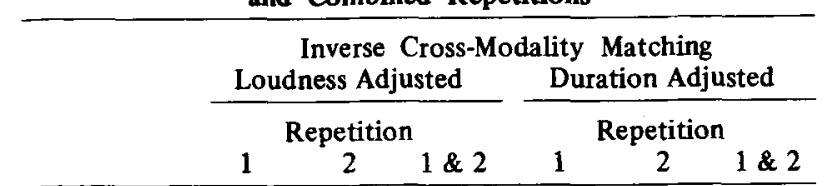

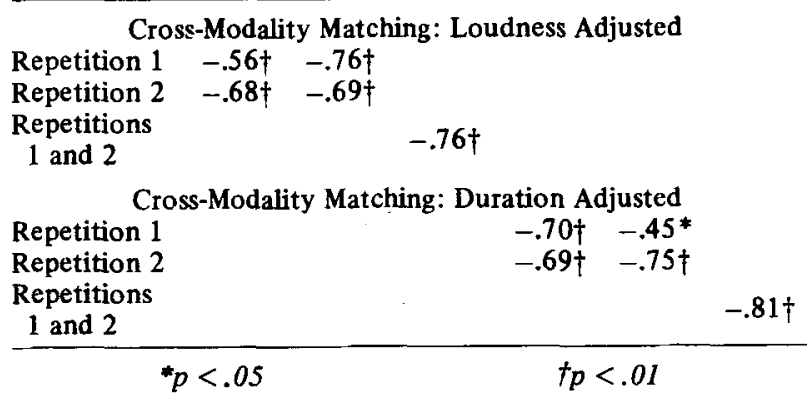

can be seen in Table 2, in which the individual exponents pooled over repetitions for each task are presented. It is compatible with Equations 1 and 2, which indicate that one exponent should equal $-(\mathrm{n} / \mathrm{m})$ while the other should equal $\mathrm{n} / \mathrm{m}$. The consistency of subjects' idiosyncratic exponents is borne out by a correlational analysis comparing direct with inverse exponents for each modality adjustment. Table 3 shows significant negative correlations for individual repetitions as well as for combined repetitions. Whatever manipulations the subject performs in the regular cross-modality task appear to be repeated in an approximately inverse manner in the inverse tasks.
Equations 1 and 2 suggest that the absolute values for cross-modality exponents should equal those for inverse matching for each modality. Therefore, the absolute values of the individual exponents were compared in a three-way repeated measures analysis of variance. As expected, there was a significant difference in exponents when different modalities were adjusted by the subject, $F(1,19)=31.32$. $p<.001$, but there was no difference between the absolute values of exponents for direct and inverse matches. The repetitions effect was also nonsignificant.

To see if the data for individuals possessed ratio consistency, the Kenney procedure was used to fit the inverse matches vs. the direct matches for each subject. As can be seen in Table 4, many of the subjects approached the -1.00 prediction quite closely, but many departed widely from this value. In addition. departure from linearity is evident for some subjects as indicated by low $r^{2}$ results. Several subjects' data were chosen at random to give an indication of the degree of curvilinearity present for individual subjects. Figures 4 and 5 illustrate that the quadratic trend evident in the group data seems to occur in varying degrees in the individual plots.

\section{Individual Differences in Exponents}

Interrepetition correlations were calculated for both types of matches in order to determine whether there were reliable individual differences in exponents (see Table 5). Correlations for direct cross-modality matching were significant, as expected on the basis of

\section{Table 4}

Exponents and Squared Correlation Coefficients for the Inverse Cross-Modality Matches Plotted as a Function of Cross-Modality Matches

\begin{tabular}{ccccl}
\hline Subject & $\begin{array}{c}\text { Loudness } \\
\text { Adjusted }\end{array}$ & $\mathrm{r}^{2}$ & $\begin{array}{c}\text { Duration } \\
\text { Adjusted }\end{array}$ & $\mathrm{r}^{2}$ \\
\hline 1 & -1.37 & .91 & -.89 & .99 \\
2 & -.84 & .96 & -.76 & .89 \\
3 & -1.08 & .96 & -.85 & .97 \\
4 & -1.00 & .98 & -.81 & .89 \\
5 & -.95 & .97 & -.86 & .87 \\
6 & -1.04 & .70 & -.93 & .93 \\
7 & -.70 & .99 & -1.20 & .93 \\
8 & -1.35 & .95 & -1.57 & .88 \\
9 & -.54 & .92 & -1.02 & .85 \\
10 & -1.04 & .81 & -.86 & .87 \\
11 & -.70 & .98 & -.92 & .80 \\
12 & -.77 & .92 & -1.07 & .91 \\
13 & -.82 & .91 & -1.04 & .94 \\
14 & -.96 & .97 & -.59 & .88 \\
15 & -1.06 & .90 & -.98 & .89 \\
16 & -1.31 & .89 & -.78 & .87 \\
17 & -.94 & .99 & -.77 & .97 \\
18 & -.92 & .89 & -1.39 & .97 \\
19 & -.98 & .98 & -.67 & .93 \\
20 & -1.92 & .82 & -.98 & .95 \\
& -1.01 & .920 & -.947 & .909 \\
Mean & .302 & .073 & .232 & .047 \\
\hline SD & & & & \\
\hline
\end{tabular}




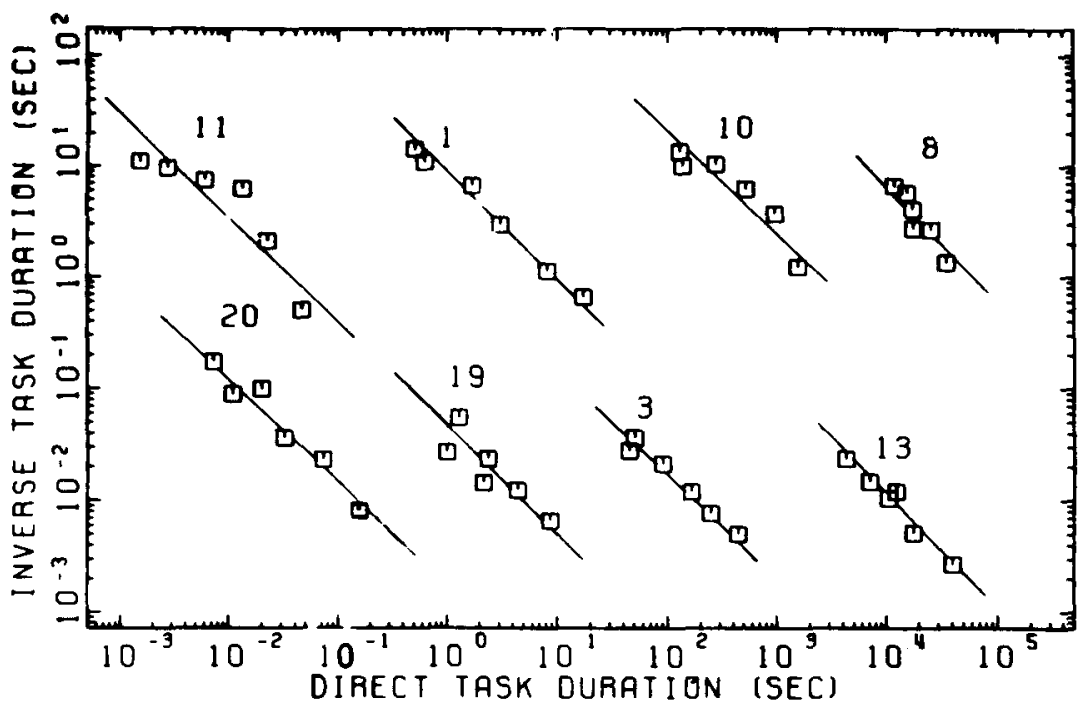

Figure 4. Inverse cross-modality matches of apparent duration to loudness as a function of crossmodality matches of apparent duration to loudness for eight randomly chosen subjects each pooled over repetitions. (Axes are rêlive with curves and datum points for subjects shifted to facilitate visual presenation.)

previous research (Rule \& Markley; 1971). Significant correlations of about the same magnitude were found for the inverse matching tasks, indicating that reliable subject differences occurred in the latter tasks also. In an attempt to compensate for these sources of variation, regression balances were performed using each subject's loudness and duration exponents, first for the first repetition and then for the second repetition. As Table 5 shows, interrepetition correlations for the balanced exponents did not show a reduction in magnitude compared to the prebalance correlations for either the direct or the inverse matches. It appears that differences in the regression effect may account for very little of the interindividual variation contrary to the speculation of Stevens (1971).

If subjects' inverse matches were like their direct matches except that their exponents were now negative, then it would be expected that any empirically obtained difference in the absolute size of the exponents for the two matches would be random. If such differences are random, then the differences for the first repetition would not presumably be correlated with those for the second repetition. Interrepetition correlations for this measure were $\mathbf{0 . 1 0}$ for duration matching and -0.07 for loudness matching, suggesting that no reliable individual differences in exponent differences occurred. This finding supports the idea that individuals are capable of a consistent inverse match, but that random factors may obscure an otherwise inversely proportional relationship.

\section{DISCUSSION}

\section{Ratio Consistency and the Power Law}

The consistency of ratio judgment has been
Figure 5. Inverse cross-modality matches of apparent loudness to duration as a function of cross-modality matches of apparent loudness to duration for eight randomly chosen subjects each pooled over repetitions. (Axes are relative with curves and datum points for subjects shifted to facilitate visual presentation.)

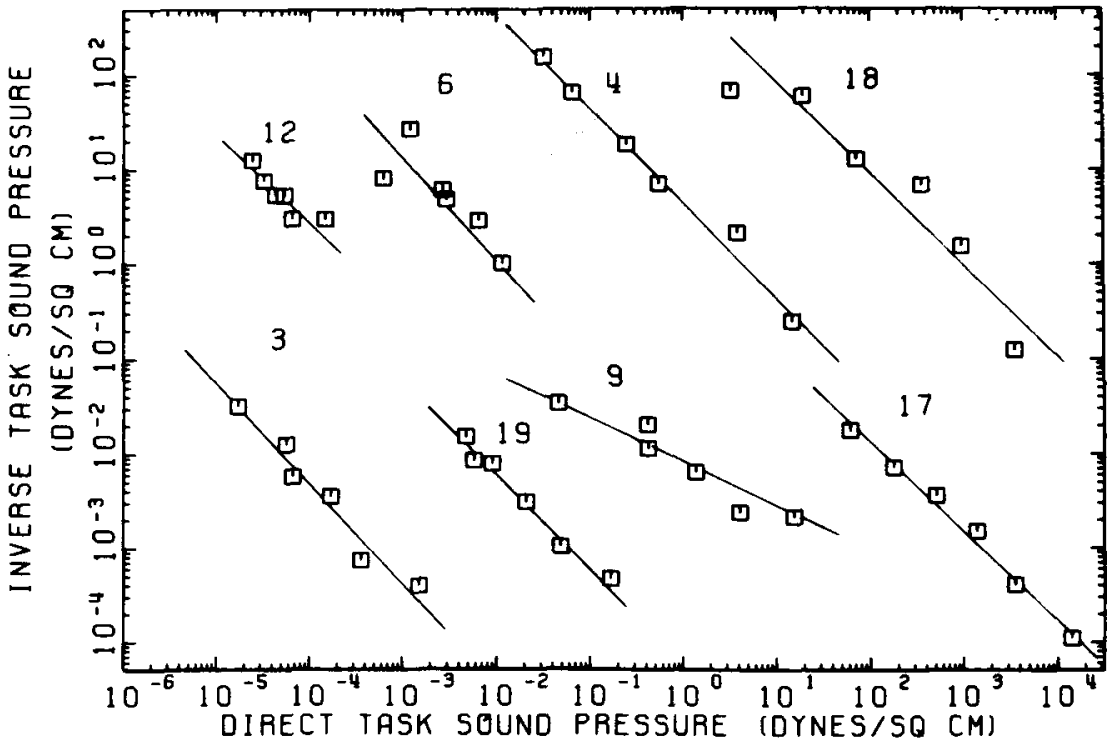


Table 5

Interrepetition Correlations for Each Task

\begin{tabular}{|c|c|c|c|c|}
\hline & \multicolumn{2}{|c|}{$\begin{array}{c}\text { Direct } \\
\text { Repetition } 2 \\
\end{array}$} & \multicolumn{2}{|c|}{$\begin{array}{c}\begin{array}{c}\text { Inverse } \\
\text { Repetition } 2\end{array} \\
\end{array}$} \\
\hline & $\begin{array}{c}\text { Loud- } \\
\text { ness }\end{array}$ & $\begin{array}{c}\text { Dura- } \\
\text { tion }\end{array}$ & $\begin{array}{c}\text { Loud } \\
\text { ness }\end{array}$ & $\begin{array}{c}\text { Dura- } \\
\text { tion }\end{array}$ \\
\hline $\begin{array}{l}\text { Direct Repetition } 1 \\
\text { Loudness Adjusted } \\
\text { Duration Adjusted } \\
\text { Balanced Exponent }\end{array}$ & $.77^{*}$ & $.61^{*}$ & & \\
\hline $\begin{array}{l}\text { Inverse Repetition } 1 \\
\text { Loudness Adjusted } \\
\text { Duration Adjusted } \\
\text { Balanced Exponent }\end{array}$ & & & $.57^{*}$ & $.57^{*}$ \\
\hline
\end{tabular}

${ }^{*} p<.01$

assessed in several ways. Among these ways have been the scaling of inverse attributes (discussed later), the comparison of multiple and fractional judgments (the reciprocal asymmetry examined by Svenson and Akesson, Note 1), and the check for multiplicative transitivity (Fagot \& Stewart, 1969). What has emerged from these studies is that (1) inverse attribute scales tend to be reciprocals of their counterpart scales, but that some evidence suggests they are not perfect reciprocals; (2) multiple and fractional judgments are nearly reciprocally related for group data and for some individuals, but that many individuals do not show reciprocal asymmetry; and (3) multiplicative transitivity does not usually hold for group or individual subject data (Fagot \& Stewart, 1969).

Inverse cross-modality matching provides a different technique for the assessment of consistency. The present research found that this task led to data that is, to a first approximation, described by a power function having an exponent equal in magnitude but different in sign to that for direct cross-modality matching. Thus, the inverted matches approximated the predictions derived from Stevens' power law. But some degree of curvilinearity was present in these results-a statistically significant amount in the case of the loudness task. Dawes (1972) has posed the question as to how consistent ratio estimates must be in order to serve as the basis for measurement. He commented that: "There is no answer that would currently be accepted by everyone using these estimates. At one extreme, Stevens and his followers tend to regard any inconsistency as random error and accept the idea that people can make such estimates meaningfully-even if they conflict with one another. The human being is treated, in effect, like a scale with a worn spring; occasional inconsistencies are to be expected; they are from imperfections in the measuring instrument instead of from any basic problem in the conceptualization. At the other extreme, investigators design experiments specifically to challenge some of the implications of the direct estimate measurement techniques; if these implications are successfully challenged, the investigator concludes that the measurement technique is invalid -at least in the context studied." The inverse matches approach the consistency Dawes discusses; however, the deviation from strict linearity points to a lack of perfect consistency in the group data. The source of the curvature remains to be identified.

\section{Individual Ratio Consistency}

According to the predictions, a subject, if he is consistent and if he follows Stevens' law, should produce the same exponent, except for sign, for both direct and inverse matches. Although subjects differed as to exponents (see Table 2), some were quite consistent across the two types of tasks, while many others were far from consistent. Consequently, when the results of inverse matches were plotted as functions of direct matches, slopes of 1.0 were found in many cases, but for some subjects the slopes departed widely from this value. In this regard, inverse matching gives results like those reported by Svenson and A kesson rather than Fagot and Stewart: group results show a first-order consistency, some individuals show it also, but some subjects are clearly inconsistent. Individuals also varied in the amount of nonlinearity present in their plots, as can be seen in Figures 4 and 5. Fagot and Stewart, though for a different type of consistency, found neither group nor individuals to be consistent.

The fact that the group and many individuals show consistency can be interpreted in different ways. Svenson and Akesson conclude that group data is suspect when many subjects are not represented by the average finding. Another possible interpretation is that the group finding is appropriate but that some subjects give atypical results due to other factors. For example, they may not understand the instructions properly, or they may be influenced by the order of the tasks or of the stimuli (cf. Cross, 1973). Possibly, such effects would be lessened with more highly practiced subjects. The present study employed subjects who were naive, at least at the outset.

\section{Individual Differences}

The present study is the first to examine scales obtained by inverse matching, and, hence, it is the first to look at such scales for the individual. In passing, it might be noted that even studies of inverse attribute scales, which have been examined more thoroughly, have rarely looked at the scales for individuals. Individual differences in exponents, indicated by the significant interrepetition correlations, were found in this study for inverse matching just as they have been found for direct matching by others (Jones \& Marcus, 1961; Rule, 1966; Rule \& Markley, 1971). Such correlations have led Rule to suggest that different persons tend to use idiosyncratic 
ranges of the matching continuum. Since the inverse and direct exponents for individuals are significantly correlated as well, the present study would support his explanation of individual differences. In fact, it adds a new situation in which they occur: previous studies found them when the same response continuum was used with different stimulus continua and when different response continua were matched to the same stimulus continuum. Now, we have a case where the stimulus and response continua remain the same while the matching instructions differ.

Although significant differences in individual exponents were found, the significant correlations across repetitions disappeared when the differences in the absolute values of the individual exponents for direct and inverse matches were compared. This suggests that whatever the subject does in the direct task is also done in the inverse task. Thus, the unique component due to the individual is approximately equal in the two tasks and through this manipulation cancels out. This finding also implies that individual ratio consistency might have occurred for more subjects were it not for random factors. Systematic effects, including persisting individual differences, were ruled out by the nonsignificant correlations.

The balance for regression was less successful. Balanced exponents for both the inverse and direct matches still yielded significant interrepetition correlations which indicates the presence of individual differences. This finding joins that of Dawson and Mirando (1975) and Wanschura and Dawson (1974) in indicating that the regression procedures suggested by S. S. Stevens (1971) are not adequate methods for the balancing out of individual differences. The new procedure, inverse cross-modality matching, seems to have some of the same difficulties and biases found in the other direct scaling techniques.

\section{Relation to Inverse Attribute Scales}

The inverse task asks the subject to match the same attribute but in an inversely proportional manner, while the inverse attribute task requests him to make adjustments in terms of the inverse attribute. Most of the studies employing the latter task have found a tendency for the inverse scale to be nonlinearly related to its corresponding primary scale. Curvature has been less strong in some experiments (S. S. Stevens \& Guirao, 1962, 1963; Stevens \& Harris, 1962) while very apparent in others (Eisler, 1962; John, 1971; Torgerson, 1960). John demonstrated by statistical analysis that his softness function was curvilinear in log-log coordinates.

The inverse cross-modality matching procedure does not lead to a more accurate and precise reciprocal relationship. Significant curvilinearity occurs with it also, although it appears to be of the less strong variety. The cause of this nonlinearity, both in inverse matching and in the inverse attribute task, remains to be identified. The present study demonstrates that the curvature is not peculiar to those tasks in which the observer is asked to judge an inverse attribute.

\section{Significance for the Power Law}

As we have noted, the results are only partially supportive of the power law. Group data, except for some curvilinearity, tend to be in line with the law, whereas the data for many individuals are clearly at odds with it. Whether the discrepancies reflect an inadequacy of the law or the general quality of the present efforts to scale deserves some consideration. Finding some curvilinearity with group data tends to be more the rule than the exception in studies of inverse attributes. This result has shown up for many investigators including those who have employed practiced and naive observers, different sensory modalities, and procedural variations such as a free vs. a fixed modulus. It appears to be a well-established embarassment to the power law which still needs explanation.

The discrepancies for individual subjects-are less easy to deal with since few of the earlier studies have examined the behavior of individual subjects and there are less data to draw upon. The fact that some persons exhibit very close to inverse behavior while others do not leads to the speculation that different subjects may be executing the task in qualitatively different manners. For example, the former persons may be actively employing numerical mediation and taking the appropriate reciprocals in the course of their matches. The latter, on the other hand, may be approaching their matches in a less mediated and more "intuitive" manner. Failure to grasp the instructions, one of the disadvantages of naive observers, also may be a source of discrepancy. As is well known, a major problem with so-called direct scaling has to do with knowing whether the subject is or is not following the instructions in the manner intended.

In the broader context of psychophysics, it should be mentioned that the results are, even with the discrepancies noted, much more in line with Stevens' power law than they are with Fechner's logarithmic law. If the derivation at the beginning of this paper were begun using two logarithmic functions rather than two power functions, a totally different prediction would ensure. Fechner's law implies that the inverse match should be related to the direct match by a hyperbolic-shaped arc, rather than a straight line with a negative unit slope, when the data are plotted on log-log axes. Clearly, the experimental results, both in the present study and in earlier inverse attribute studies, are not of this shape. When curvature has been present, it has been in the opposite direction. 


\section{REFERENCE NOTE}

1. Svenson, O., \& Akesson, C. A. Fractional and multiple estimates in ratio scaling. Reports from the Psychological Laboratories, University of Stockholm, No. 202, January 1966.

\section{REFERENCES}

Cliff. N. Scaling. Annual Review of Psychology, 1973, 24, 473-506.

Cross, D. V. Sequential dependencies and regression in psychophysical judgments. Perception \& Psychophysics, 1973, 14. 547.552 .

DAwEs. R. M. Fundamentals of attitude measurement. New York: Wiley, 1972.

Dawson, W. E., \& Mirando, M. A. Sensory-modality opinion scales for individual subjects. Perception \& Psychophysics, 1975. 17. 596-600.

EISLER. H. Empirical test of a model relating magnitude and category scales. Scandinavian Joumal of Psychology, 1962, 3. 88-96.

Ekman, G., Hosman, B., Lindman, R., Lunngberg, L., \& AxEsson, C. Interindividual differences in scaling performance. Perceptual and Motor Skills, 1968, 26, 815-823.

Fagot, R. F., \& STEwart, M. Tests of product and additive scaling axioms. Perception \& Psychophysics, 1969, 5, 117-123.

JonN, I. D. The properties of distributions of magnitude estimates of loudness and softness. Scandinavian Joumal of Psychology, 1971, 12, 261-270.

Jones, F. N., \& MArcus, M. J. The subject effect in judgments of subjective magnitude. Journal of Experimental Psychology, $1961,61,40-44$.

Kenney, J. F. Mathematics of statistics (Part one). New York: Van Nostrand, 1939.

Poulton, E. C., \& Stevens, S. S. On the halving and doubling of the loudness of white noise. Journal of the Acoustical Society of America, 1955, 27, 329-331.

RULE, S. Subject differences in exponents of psychophysical power functions. Perceptual and Motor Skills, 1966, 23, 1125-1126.
Rule, S., \& Markley, R. P. Subject differences in crossmodality matching. Perception \& Psychophysics, 1971, 9, 115-117.

Stevens, J. C., \& Macx, J. D. Scales of apparent force. Journal of Experimental Psychology, 1959, 58, 405-413.

STEvens, S. S. The measurement of loudness. Journal of the Acoustical Society of America, 1955, 27, 815-829.

Stevens, S. S. On the psychophysical law. Psychological Review, $1957,64,153-181$.

Stevens, S. S. The psychophysics of sensory function. In W. A. Rosenblith (Ed.), Sensory communication. New York: Wiley, 1961.

STEvens, S. S. On prediction of exponents for cross-modality matches. Perception \& Psychophysics, 1969, 6, 251-256.

STEvens, S. S. Issues in psychophysical measurement. Psychological Review, 1971, 78, 426-450.

Stevens. S. S., \& Greenbaum, H. B. Regression effects in psychophysical judgment. Perception \& Psychophysics, 1966, 1, 439-446.

Stevens, S. S., \& Gurrao, M. Loudness, reciprocality, and partition scales. Joumal of the Acoustical Society of America, $1962,34,1466-1471$.

Stevens, S. S., \& Guirıo, M. Subjective scaling of length and area and the matching of length to loudness and brightness. Journal of Experimental Psychology, 1963, 66, 177-186.

Stevens. S. S., \& Harris, J. R. The scaling of subjective roughness and smoothness. Joumal of Experimental Psychology, $1962,64,489-494$.

Torgerson, W. S. Quantitative judgment scales. In H. Gulliksen \& S. Messick (Eds.), Psychological scaling: Theory and applications. New York: Wiley, 1960.

Wanschura, R. G., \& Dawson, W. E. Regression effect and individual power functions over sessions. Journal of Experimental Psychology. 1974, 102, 806-812.

(Received for publication July 28, 1975; revision received October 16,1975 .) 